\title{
Contribuições de Estudos de Caso para o Avanço do Conhecimento sobre Superdotação
}

\author{
Eunice M. L. Soriano de Alencar \\ Universidade de Brasília - Brasília - DF
}

\section{Resumo}

Muitos foram os avanços na compreensão das distintas facetas da superdotação. Estes avanços resultaram de pesquisas, utilizando-se numerosas metodologias. Entretanto, constata-se um número muitíssimo reduzido de estudos de caso publicados, apesar de sua adequação para investigação de indivíduos com desempenho ou habilidades excepcionais. Ademais, múltiplos estudos de caso de um grupo diferenciado permitem desenvolver teorias para explicar o fenômeno, em sua complexidade e riqueza. Para ilustrar possíveis contribuições desses estudos, são apresentadas duas pesquisas, reconhecidas por expandirem o conhecimento da área. A primeira, desenvolvida por Feldman com uma amostra de crianças prodígios, que redundou na proposição da teoria da co-incidência. A segunda, realizada por Reis, com mulheres eminentes, que resultou na construção da teoria do desenvolvimento do talento feminino. A primeira teoria inclui distintos fatores que se associam ao surgimento de prodígios e a segunda, às condições que possibilitam realizações excepcionais em mulheres, elementos esses anteriormente pouco conhecidos.

Palavras-chave: Estudo de caso; superdotados; talento.

\section{Contributions Case Studies for the Advancement of Knowledge on Giftedness}

\begin{abstract}
There have been many advances in the understanding of the different facets of giftedness. These advances have resulted from research using numerous methods. However, there has been a greatly reduced number of published case studies, despite its suitability for investigation of individuals with exceptional abilities or performance. In addition, multiple case studies from a different group allow developing theories to explain the phenomenon in its complexity and richness. To illustrate this, two investigations are presented, they also are, broadly recognized for having expanded the knowledge of the area. The first, developed by Feldman with a sample of child prodigies, which resulted in the proposition of the theory of co-incidence. The second, developed by Reis, with eminent women, which resulted in the construction of the theory of female talent development. The first theory includes different factors associated to the emergence of wonders and the second, the conditions that enable exceptional achievements in women, these previously little known elements.
\end{abstract}

Keywords: Case study; gifted; talent.

\section{Contribuciones de Estudios de Caso para el Avance del Conocimiento sobre Superdotación}

\begin{abstract}
Resumen
Muchos han sido los avances en la comprensión de las distintas facetas de la superdotación. Estos avances resultaron de investigaciones, utilizándose numerosas metodologías. Sin embargo, se percibe un número muchísimo reducido de estudios de caso publicados, pese su adecuación para investigación de individuos con desempeño o habilidades excepcionales. Además, múltiples estudios de caso de un grupo diferenciado permiten desarrollar teorías para explicar el fenómeno, en su complexidad y riqueza. Para ilustrar posibles contribuciones de esos estudios, son presentadas dos investigaciones, reconocidas por expandir el conocimiento del área. La primera, desarrollada por Feldman con una muestra de niños prodigios, que resultó en la proposición de la teoría de la co-incidencia. La segunda, realizada por Reis, con mujeres eminentes, que resultó en la construcción de la teoría del desarrollo del talento femenino. La primera teoría incluye distintos factores que se asocian al surgimiento de prodigios y la segunda, a las condiciones que posibilitan realizaciones excepcionales en mujeres, elementos esos anteriormente poco conocidos.
\end{abstract}

Palabras clave: estudio de caso; superdotados; talento. 


\section{Introdução}

Muitos foram os avanços observados na compreensão das diversas facetas da superdotação'. Provas desses avanços e novas tendências em pesquisa e prática encontram-se em numerosos livros e periódicos que surgiram nestas últimas décadas, focalizando tópicos pouco conhecidos e discutidos há muito tempo, como, por exemplo, características neuropsicológicas da superdotação, dupla excepcionalidade e metacognição em indivíduos superdotados. Uma dessas publicações é o International Handbook on Giftedness, editado por Shavinina (2009a), com mais de 1500 páginas e capítulos redigidos por eminentes pesquisadores, abordando tópicos diversificados relativos à superdotação e suas distintas expressões, natureza, fatores que contribuem ou dificultam a realização mais plena do potencial superior. Um exame das principais publicações da área sinaliza, entretanto, um número muito reduzido de pesquisas utilizando como método de coleta de dados o estudo de caso, apesar de ser este especialmente adequado para a investigação e descrição de indivíduos que se destacam por um desempenho extraordinário ou habilidade excepcional (Foster, 1986; Gross, 2000). Na análise, por exemplo, dos artigos publicados em Gifted Child Quarterly, dos últimos cinco anos, encontraram-se apenas três estudos de caso (Assouline, Nicpon, \& Doobay, 2009; Lee, \& Sriraman, 2012; Ng, \& Nicholas, 2010), ao passo que nenhum estudo desta natureza com indivíduos superdotados/talentosos foi publicado na Gifted Educational International nos últimos 10 anos.

Uma breve descrição da literatura referente a estudos de caso de um único indivíduo com um nível extremamente elevado de habilidade é apresentada por Gross (2005). Essa autora, que investigou com profundidade 15 crianças com um QI excepcionalmente elevado (acima de 160), acompanhando-as ao longo de vários anos, chama a atenção para o reduzido número de pesquisas referentes a estudos de caso de crianças com níveis fenomenais de habilidade, ressaltando ainda que estudos de caso, de caráter longitudinal, são muito raros. Gross lembra também as vantagens e desvantagens dessa modalidade de pesquisa. Como vantagens, aponta poder se alcançar uma visão holística do indivíduo por meio de dados levantados com uma variedade de procedimentos, como observação, entrevistas e documentos; a sua utilidade de uso quando não é factível ou desejável manipular causas potenciais do comportamento; e ainda a possibilidade de desenvolver e validar teorias a partir de múltiplos estudos de caso de um grupo diferenciado de indivíduos, o que contribui de forma expressiva para explicar a natureza do fenômeno, em sua complexidade e riqueza. Como limitações, Gross ressalta os altos investimentos em termos de tempo do pesquisador, notadamente quando se trata de múltiplos estudos

1 Superdotação é um fenômeno multifacetado, para o qual não existe uma definição precisa e aceita universalmente. $O$ termo tem sido utilizado para se referir a indivíduos que apresentam uma performance consistentemente superior em uma linha de atividade humana potencialmente de valor (Passow, Mönks, \& Heller, 2010). de caso de indivíduos que residem em diferentes cidades, além dos recursos financeiros necessários para tal.

Entre os estudos de caso de caráter múltiplo, ou seja, de um grupo em particular de indivíduos com características similares, mais conhecidos e referenciados na literatura de superdotação, poder-se-iam apontar o realizado por Hollingworth (1942) com 12 crianças (oito meninos e quatro meninas) que apresentavam um QI acima de 180 na Escala de Inteligência Starford-Binet e o desenvolvido por Gross (2005), citado anteriormente, que documentou o desenvolvimento acadêmico, social e emocional de 15 crianças (10 do sexo masculino e 5 do sexo feminino) com um QI acima de 160, o de Morelock (Morelock, \& Feldman, 2000, 2003) com oito crianças (sete meninos e uma menina), com um Q। extraordinariamente elevado (seis delas apresentavam um QI bem acima de 200). Também podemos citar o estudo de Feldman (1991, 2012; Morelock, \& Feldman, 2000, 2003) com seis crianças prodígios do sexo masculino, e ainda o realizado por Reis (1998, 2002, 2005; Reis \& Sullivan, 2009), com 22 mulheres norte-americanas, com expressiva projeção nos mais diversos domínios. Para ilustrar as possíveis contribuições desse tipo de investigação, foram selecionadas duas pesquisas, entre as anteriormente citadas. Os critérios para essa escolha foram: (a) apresentar múltiplos estudos de caso, envolvendo vários participantes; (b) ter gerado teorias para explicar o fenômeno investigado.

A primeira, desenvolvida por David Feldman com uma amostra de seis crianças prodígios, levou o autor a propor a teoria da co-incidência, que engloba diversos vetores, com valor heurístico para orientar novos estudos sobre dimensões relevantes da superdotação e criatividade (Feldman, 1990, 1991, 1992, 2012). A segunda, realizada por Sally Reis com 22 mulheres norte-americanas eminentes, com dados levantados ao longo de duas décadas, resultou na construção da teoria do desenvolvimento do talento feminino, que inclui também distintos fatores que se associam a uma expressão maior do talento em mulheres. São estudos que merecem ser mais divulgados e conhecidos, dadas as suas implicações para o desenvolvimento do potencial superior, uma vez que sinalizam um conjunto de condições que se relacionam com tal desenvolvimento.

Ressalta-se que, no Brasil, a literatura registra vários estudos de caso de indivíduos superdotados, como os desenvolvidos por Alencar e Fleith (2001), Cohen (2013), Ferreira (2013) e Pérez e Freitas (2013). Entretanto, desconhecemos pesquisas realizadas no país que atendessem aos critérios propostos para seleção das investigações a serem descritas. Ademais, múltiplos estudos de caso que tenham gerado teorias são em número muitíssimo reduzido. Foi esta a razão de limitarmos a apenas duas as pesquisas aqui apresentadas.

\section{O Estudo de Feldman com uma Amostra de Crianças Prodígios e a Teoria da Co-Incidência}

Como apontamos anteriormente em texto de nossa autoria (Alencar, \& Fleith, 2001), o interesse por crianças 
com um desempenho extraordinário em um domínio valorizado socialmente tem sido registrado desde tempos imemoriais. Há registros, por exemplo, de crianças chinesas, que viveram em distintas dinastias, e que surpreendiam a todos por sua extrema precocidade e performance excepcional. Uma análise desses registros foi realizada por Kwok (s.d.), que faz referência a diversas crianças, como Li Bai, famoso poeta chinês, que memorizou a obra clássica de Confúcio aos cinco anos. Outro exemplo citado é Quan De Yu que, desde os quatro anos, escrevia poesias que eram motivo de encanto por parte daqueles que o conheciam. Lembra Kwok que, na China, durante várias dinastias, prevaleceu um interesse em identificar crianças com habilidades excepcionais, havendo mesmo um exame e um sistema de premiação para os prodígios colocados em prática desde o século VII. Essas crianças eram chamadas de shen tong ou crianças com habilidades supernaturais.

Morelock e Feldman (2003) também apresentam vários exemplos de extrema precocidade registrados ao longo dos séculos, ao passo que Shavinina (2009b) descreve algumas crianças prodígios investigadas por Leites (citado por Shavinina, 2009b) desde o final dos anos 1950, na então União Soviética, e cujas características encontram-se em livros publicados em Moscou. Segundo Shavinina, para Leites, o fenômeno do prodígio poderia ser explicado por elevada atividade mental (habilidade de se dedicar a trabalhos mentais por longos períodos de tempo, necessidade permanente de atividade intelectual, curiosidade excepcional, uma ampla variedade de interesses), ótimo funcionamento da autorregulação (processos de metacognição responsáveis pelo planejamento, monitoramento e controle executivo), além do que denomina sensitividade relacionada à idade (age sensitivity), definida como "responsividade específica, intensa e muito seletiva de um indivíduo a tudo que esteja ao seu redor" (Shavinina, 2009b, p. 237). Mais recentemente, com os avanços do conhecimento sobre a neuropsicologia da superdotação, novas hipóteses surgiram para explicar a dinâmica fundamental de crianças prodígios. Uma dessas hipóteses foi proposta por Vanderbelt (2009), pesquisador que tem investigado a base neurofisiológica da criatividade e inovação (Vanderbelt, 2003; Vanderbelt, Schimpf, \& Liu, 2007). Esse autor propõe que:

o desempenho extraordinário de crianças prodígios é o resultado de controle de alta atenção em um domínio específico aprendido bem cedo na infância e constantemente modulado entre o córtex pré-frontal e as funções cognitivas modeladoras do cerebelo. Conclui-se que este controle de alta atenção em crianças prodígios acelera a produção de processos intelectuais elevados em uma versão espontânea de prática deliberada (p. 295).

Entretanto, é o estudo de Feldman (1991), descrito a seguir, o que talvez mais tenha contribuído para chamar a atenção para numerosos fatores referentes tanto ao indivíduo quanto ao ambiente mais próximo e mais remoto, que favorecem o desenvolvimento excepcional do potencial humano, característico de crianças prodígios. Feldman (1990, p. 209) define prodígio como "uma criança que é capaz de apresentar um desempenho igual ou similar ao de um adulto profissional em um campo intelectualmente exigente". A sua pesquisa teve início em 1975 com três crianças prodígios sendo incorporadas à pesquisa e mais três em 1978, todas elas de classe média ou média alta. Visitas e entrevistas com as crianças, que eram todas do sexo masculino, seus pais, professores, irmãos, amigos e colegas de sala, além de especialistas nos campos em que essas se destacavam, foram feitas por Feldman, ao longo de vários anos, algumas delas entrevistadas dezenas de vezes e outras cinco ou seis vezes (Feldman, 1991). Entre elas, havia dois jogadores de xadrez, que, com menos de 10 anos de idade, que se encontravam entre os meIhores dos Estados Unidos; um músico/compositor, cujo forte interesse por música se manifestou antes de completar três anos; um matemático, que iniciou os estudos de álgebra nos primeiros anos de escola; um escritor, que redigia histórias e peças teatrais antes de completar cinco anos; e um prodígio, a que denominou de omnibus, que apresentava um desempenho excepcional em uma variedade de áreas, entre elas, composição musical. Era interesse de Feldman (1991, 1992, 2012) observar, compreender e explicar o fenômeno prodígio, tendo as suas observações culminado com a construção de uma teoria, a qual chamou de teoria da co-incidência e que inclui distintas facetas, especificadas a seguir (Feldman, 1992, 2012; Morelock, \& Feldman, 1993, 2003):

- Qualidades biológicas - fatores genéticos e não-genéticos que formam o substrato para $o$ desenvolvimento de talentos diferenciais ou capacidade intelectual geral.

- Qualidades psicológicas individuais - habilidades distintas de natureza intelectual, como funções executivas e de planejamento; e qualidades pessoais e emocionais, como dedicação apaixonada ao campo em que se destaca por seu desempenho extraordinário e hipersensibilidade (sensibilidades inusitadas, que se refletem nas reações do indivíduo a experiências sensoriais ou intelectuais seletivas).

- Contexto próximo - ambiente imediato do desenvolvimento da criança, incluindo fatores, como higiene, nutrição e intervenções educacionais organizadas por professores que instruem o prodígio, mantendo a sua dedicação e comprometimento à área de seu interesse, seja música, xadrez ou artes gráficas.

- Contexto intermediário - esta dimensão inclui, entre outros, a estrutura familiar, número de irmãos, ordem de nascimento, tradições familiares, como, por exemplo, histórias de interesse especial em um domínio específico, que podem servir para orientar a criança para um domínio particular. 
- Domínio e campo em que a criança apresenta comportamentos prodigiosos, o primeiro com suas formas correntes e emergentes, técnicas e tecnologias para expressão e exploração de formas de manifestação do domínio; e o segundo, que medeia o contato entre a pessoa e o domínio, incluindo professores, mentores, academias, comissões e audiências que sustentam e dirigem a atividade, associando-se à forma como o talento é reconhecido, recrutado e desenvolvido.

- Distintos contextos - esta dimensão inclui circunstâncias históricas e sociais, que encorajam, reprimem ou modelam a expressão do potencial individual. Movimentos políticos, instituições religiosas, eventos, como guerra e recessões, são exemplos de fatores que contribuem para o desenvolvimento e expressão, em maior ou menor grau, do potencial humano.

No livro de sua autoria, Feldman (1991) descreve detalhadamente cada um dos prodígios e chama a atenção para o papel crucial da família como catalizadora e organizadora da co-incidência. Descreve como a família respondeu ao tomar consciência do talento extremo do filho e os procedimentos utilizados para encorajar e facilitar o desenvolvimento do potencial da criança, não medindo esforços para contratar, por exemplo, professores e localizar recursos que pudessem colaborar para o desempenho extraordinário do filho. Discorre também sobre os domínios que produzem prodígios, ressaltando que alguns campos do conhecimento, mais do que outros, oferecem um terreno fértil para o aparecimento de prodígios. Lembra, por exemplo, que, em alguns domínios, como música e xadrez, indivíduos muito jovens podem alcançar níveis mais elevados em um período de tempo inusitadamente curto, que usualmente exigira uma década ou mais de estudos. Esses seriam os domínios que permitem o surgimento de prodígios. Alguns outros, como a filosofia, dependem muito mais de experiência do que conhecimento específico ou técnica. Esta seria uma das razões para o maior número de prodígios observados na performance musical e no desempenho em xadrez do que em outros domínios

A combinação de fatores pessoais e culturais é enfatizada por Feldman, com base nos dados por ele coletados. Destaca o autor:

O fenômeno da criança prodígio é o resultado de uma concordância fortuita de forças que coincidem levando a uma maximização do potencial humano. Essencialmente, na maior parte dos casos, uma criança com habilidade extraordinária surge em uma família que reconhece, valoriza e cultiva tal habilidade (...) A criança é geralmente exposta a professores de melhor formação que instruem o prodígio de uma maneira que favorece a manutenção do seu interesse e dedicação. Invariavelmente, a criança demonstra uma combinação de autodireção e dedicação apaixonada por sua área de realização. (Morelock, \& Feldman, 2000, p. 228).

\section{O Estudo de Reis com Mulheres Eminentes e a Teoria do Desenvolvimento do Talento Feminino}

A literatura registra várias pesquisas com mulheres que se projetaram em áreas diversas, entre elas a de Subotnik e Arnold (1995), que investigaram, por meio de entrevista, 11 mulheres com realização notável na área de ciências e a de Kerr (1994, 2011), que analisou as biografias de 33 mulheres eminentes, algumas delas que tinham vivido na segunda metade do século XIX e outras no século passado. Ambos os estudos chamam a atenção para características pessoais e fatores que influenciaram as aspirações e realizações das duas amostras investigadas. Entretanto, foi Reis (1998, 2002; Reis, \& Sullivan 2009) quem se debruçou sobre o desenvolvimento do talento feminino, realizando múltiplos estudos de caso, de caráter longitudinal, que culminaram na construção de uma nova teoria. Ressalta-se que as investigações de Reis com mulheres superdotadas/ talentosas tiveram início na década de 1970 e incluíram não apenas amostra de mulheres eminentes, mas também de adolescentes superdotadas e mulheres, com altos níveis de potencial, que abriram mão de uma vida profissional mais produtiva, reconhecida e valorizada na sociedade, para se dedicar integralmente à família ou perseguir outros interesses, como, por exemplo, de caráter comunitário.

Neste artigo será apresentada brevemente a teoria proposta por Reis (1998, 2002; Reis, \& Sullivan, 2009) para explicar o desenvolvimento do talento feminino e que resultou do estudo desenvolvido por ela com 22 mulheres americanas que se destacaram, de forma altamente expressiva, em áreas diversas (política, literatura e poesia, teatro, artes, composição musical, negócios, ciências, entre outras), sendo muitas delas a primeira ou uma das primeiras mulheres norte-americanas a alcançar alta projeção em uma área específica, como atuar como guarda florestal e ecologista. Foram feitos estudos de caso, de natureza qualitativa, com dados coletados ao longo de duas décadas, buscando-se identificar as suas características personológicas, interesses relacionados à sua carreira e outros interesses, fatores que contribuíram ou dificultaram o desenvolvimento de suas habilidades, a forma como exerciam os seus talentos, satisfação com a vida, entre outros aspectos. Utilizaram-se para tal um questionário com 22 perguntas, entrevistas em profundidade com essas mulheres ou sobre elas, além de documentos referentes às suas realizações, como publicações, diários, composições musicais e premiações. Cada uma foi entrevistada pelo menos cinco vezes durante o período de coleta de dados.

Com base na investigação da trajetória de vida pessoal e profissional destas mulheres, Reis (1998, 2005, 2013; Reis, \& Sullivan, 2009) constatou uma variedade de formas com que desenvolveram seus talentos e que este desenvolvimento havia ocorrido em distintos períodos de suas vidas, em alguns casos ao longo de várias décadas e em outros, tardiamente. Algumas delas, por exemplo, encontraram grandes dificuldades em determinadas etapas da vida para se dedicar à sua área de interesse, em função de 
diversos fatores, como expectativas e obrigações que limitavam as suas oportunidades de uma produção criativa, além de valores predominantes na sociedade norte-americana de poucas décadas atrás, que dificultavam e mesmo impediam a contratação de mulheres para determinadas funções. Reis $(2005,2013)$ verificou ainda que as experiências contextuais do grupo investigado diferiam das de homens eminentes em diversas esferas. Por exemplo, muitas dessas mulheres diversificavam as áreas em que canalizavam seus esforços criativos, como relacionamentos, família e casa, interesses pessoais, trabalhos comunitários e ainda assim produziam trabalhos de alto nível, sendo a sua criatividade produtiva muito mais difusa do que a observada em pesquisas com amostras de homens proeminentes em distintas áreas.

Foi especialmente com base neste estudo que Reis (1998, 2005, 2013; Reis \& Sullivan, 2009) propôs uma teoria do desenvolvimento do talento feminino. Esta teoria, que continua a ser refinada, segundo a autora, inclui os seguintes fatores:

- Habilidade acima da média e talentos especiais em áreas, como música, drama e redação, além de inteligência contextual ou prática, que é uma das facetas da teoria da inteligência proposta por Sternberg $(1986,1997,2005)$ e que diz respeito à facilidade de se adaptar ou mudar o ambiente de tal forma a facilitar o desenvolvimento e expressão do talento pessoal.

- Traços de personalidade - determinação, paciência no sentido, por exemplo, de esperar pela oportunidade de poder se dedicar de forma mais intensa a sua área de interesse, predisposição a correr riscos calculados e coragem para realizar tarefas relacionadas ao seu campo de atuação que, a seus olhos, outras mulheres não teriam a ousadia ou interesse em fazê-las, além de intensa concentração, foco e paixão pelo trabalho, reflexo de um altíssimo nível de motivação.

- Fatores ambientais - não há um padrão único e uniforme de ambiente que assegure o desenvolvimento do talento feminino. Os dados obtidos por Reis referentes às 22 mulheres que participaram do seu estudo indicaram que algumas eram oriundas de famílias de status socioeconômico alto e outras não; algumas estudaram em universidades de prestígio e outras sequer tinham um diploma de curso superior; a maioria foi criada em famílias que incentivavam os seus talentos; algumas enfrentaram ambientes adversos no trabalho, outras trabalhavam de forma isolada e, ainda, outras mudavam de local de trabalho com frequência; algumas tiveram o apoio de seus cônjuges e outras não. Algumas informaram que se sentiam satisfeitas com a sua vida pessoal e outras que vivenciavam períodos de infelicidade e isolamento; algumas conciliavam a vida pessoal e de trabalho sem dificuldades e outras não. Todas mantinham, entretanto, relações próximas com pelo menos uma pessoa, sejam amigos, colegas, irmãos, marido, que dava $(\mathrm{m})$ a elas o suporte necessário para se manterem ativas nos seus projetos. O trajeto para eminência foi único para cada uma das mulheres investigadas, embora todas tivessem buscado ativamente, em períodos distintos, o apoio e condições para se realizarem e se projetarem nas áreas de seu interesse.

- Percepção da importância social do trabalho realizado e sentido de destino na vida - todas as mulheres que participaram do estudo de Reis tinham consciência da importância social de seu trabalho e um desejo de fazer uma diferença no mundo por meio do uso de seus talentos. Acreditavam no impacto positivo de seu trabalho na sociedade e, por esta razão, não mediam sacrifícios para realizar o que consideravam ser o seu propósito de vida.

O estudo de Reis (1986, 2005, 2013; Reis, \& Sullivan, 2009) identificou também, de forma similar a estudos anteriores, como os realizados por Subotnik e Arnold (1995) e Kerr (1994, 2011), numerosas barreiras internas, como sentimentos de solidão e isolamento, e externas, como falta de apoio por parte da família, amigos ou colegas, que dificultam o alcance de níveis mais altos de realização. Sinaliza também como os atributos pessoais, como resiliência, determinação, energia e prazer na realização do trabalho, contribuíram na busca de estratégias por parte das mulheres que participaram do estudo, para não se deixar vencer por dificuldades enfrentadas na trajetória em direção à realização pessoal.

Reis (2005; Reis, \& Sullivan, 2009) considera que não há uma fórmula única que leve ao sucesso e sintetiza a sua perspectiva sobre o processo do desenvolvimento do talento feminino em mulheres eminentes, ressaltando:

O desenvolvimento do talento feminino ocorre quando mulheres com alta habilidade intelectual, criativa, artística ou de liderança ou potencial se destacam em uma área escolhida por elas e quando dão contribuições que consideram significativas para a sociedade, Tais contribuições são facilitadas quando as mulheres desenvolvem relações pessoais satisfatórias e buscam realizar o que acreditam ser um importante trabalho que ajuda a tornar o mundo mais saudável, mais bonito e um local de paz, no qual uma diversidade de expressões de arte e humanidade é celebrada. (Reis, 2005, p. 217).

\section{Considerações Finais}

As duas pesquisas apresentadas neste artigo ilustram estudos de caso, de caráter múltiplo, que, pela sua relevân- 
cia e riqueza de dados, muito contribuíram para expandir os conhecimentos sobre superdotação: a primeira delas sobre o fenômeno da criança prodígio e fatores associados a esse fenômeno, e a segunda para explicar o desenvolvimento do talento feminino. Ambas chamam a atenção para numerosos elementos propulsores e restritivos, tanto na família quanto no ambiente social, do desenvolvimento e expressão do potencial superior. Ambas têm sido inspiradoras para pesquisadores da área, que têm realizado estudos, seja com crianças prodígios ou com mulheres de grande destaque, com base nos pressupostos teóricos desenvolvidos por Feldman e Reis. Este foi, por exemplo, o caso da investigação de Prado (2010; Prado, \& Fleith, 2012), com uma amostra de 111 pesquisadoras brasileiras de destaque, oito das quais foram entrevistadas. Essa autora constatou, em sua amostra, vários dos elementos propostos por Reis em sua teoria do desenvolvimento do talento feminino, como, por exemplo, características individuais que refletem um alto nível de motivação, percepção da importância do trabalho realizado e circunstâncias do ambiente, algumas delas favoráveis e outras desfavoráveis, à dedicação às atividades profissionais e realização mais plena.

O presente artigo tem limitações a serem apontadas. Uma delas é ter se restringido à descrição de apenas dois estudos de caso, de caráter múltiplo, e as teorias deles resultantes. Outra é terem sido ambos desenvolvidos nos Estados Unidos. Neste sentido, considera-se relevante que, em publicação futura, sejam apresentadas pesquisas dessa natureza, com dados coletados em países europeus, asiáticos ou em outros continentes. Nessa linha de investigação, poder-se-ia também realizar um estudo comparativo de distintas teorias construídas a partir de estudos de caso múltiplos, identificando-se pontos comuns e divergentes. Ademais, sabe-se que ainda há muito a se investigar a respeito tanto de crianças prodígios quanto do desenvolvimento do talento feminino. $\mathrm{O}$ conhecimento que se tem a respeito desses dois tópicos é ainda bastante limitado. Espera-se que este artigo incite a realização de novas investigações, utilizando métodos diversos de coleta de dados, contribuindo assim para ampliar o que se sabe a respeito desses tópicos, de interesse não apenas de pesquisadores, mas também de educadores e psicólogos.

\section{Referências}

Alencar, E. M. L. S., \& Fleith, D. S. (2001). Lim: características e desenvolvimento de uma criança com uma inteligência matemática excepcional. Em E. M. L. S. Alencar (Org.), Criatividade e educação de superdotados (pp. 206-229). Petrópolis: Vozes.

Assouline, S. G., Nicpon, M. F., \& Doobay, A. (2009). Profoundly gifted girls and autism spectrum disorder: a psychometric case study comparison. Gifted Child Quarterly, 53, 239-251.

Cohen, L. M. (2013). Sementes da criatividade em dois irmãos brasileiros eminentes. Em D. S. Fleith, \& E. M. L. S. Alencar (Orgs.),
Superdotados. Trajetórias de desenvolvimento e realizações (pp. 75-94). Curitiba: Juruá.

Feldman, D. H. (1990). Giftedness as a developmentalist sees it. Em R. J. Sternberg, \& J. E. Davidson (Orgs.), Conceptions of giftedness (pp. 285- 305). Cambridge: Cambridge University Press.

Feldman, D. H. (1991). Nature`s gambit. New York: Teachers College Press.

Feldman, D. H. (1992). The theory of co-incidence: How giftedness develops in extreme and less extreme cases. Em F. Mönks, \& W. Peters (Orgs.), Talent for the future (pp. 10-22). Assen/Maastricht: Van Gorcum.

Feldman, D. H. (2012). Out of the usual course of nature: Child prodigies and their domains. Palestra proferida na 13a. Conferência Internacional do European Council for High Ability, Münster, Alemanha, setembro de 2012.

Ferreira (2013). J. F. C. (2013). Hannah, uma trajetória de superação. Em D. S. Fleith, \& E. M. L. S. Alencar (Orgs.), Superdotados. Trajetórias de desenvolvimento e realizações (pp. 41-54). Curitiba: Juruá.

Foster, W. (1986). The application of single subject research methods to the study of exceptional ability and extraordinary achievement. Gifted Child Quarterly, 30, 33-37.

Gross, M. U. M. (2000). Issues in the cognitive development of exceptionally and profoundly gifted individuals. Em K. A. Heller, F. J. Mönks, R. J. Sternberg, \& R. F. Subotnik (Orgs.), International handbook of giftedness and talent (pp. 179-182). Oxford; Elsevier.

Gross, M. U. M. (2005). Exceptionally gifted children. New York: Routledge Farmer.

Hollingworth, L. (1942). Children above 180 IQ. Origin and development. New York: World Books.

Kerr, B. A. (1994). Smart girls, gifted women. Columbus: Ohio Psychology Publications.

Kerr, B. A. (2011,). New rules for gifted girls: Lessons from NSF gender equity projects. Trabalho apresentado na 58a. Convenção da National Association for Gifted Children. New Orleans, Estados Unidos.

Kwok, C. K. (s. d.). Gifted and talented education in China: From ancient to modern times. Hong Kong: Kwok.

Lee, K. H., \& Sriraman, B. (2012). Gifted girls and nonmathematical aspirations: a longitudinal case study of two gifted Korean girls. Gifted Child Quarterly, 56, 3-14.

Morelock, M. J., \& Feldman, D. H. (1993). Prodigies and savants: What they have to tell us about giftedness and human cognition. Em K. 
A. Heller, F. J. Mönks, R. J., \& A. H. Passow (Orgs.), International handbook of research and development of giftedness and talent (pp. 161-181). Oxford; Elsevier.

Morelock, M. J., \& Feldman, D. H. (2000). Prodigies, savants and Williams Syndrome: Windows into talent and cognition. Em K. A. Heller, F. J. Mönks, R. J. Sternberg, \& R. F. Subotnik (Orgs.), International handbook of giftedness and talent (pp. 227-241). Oxford: Elsevier.

Morelock, M. J., \& Feldman, D. H. (2003). Extreme precocity: Prodigies, savants, and children of extraordinarily high IQ. Em N. Colangelo, \& G. A. Davis (Orgs.), Handbook of giftred education (pp. 455-469). Boston: Allyn and Bacon.

Ng, W., \& Nicholas, H. (2010). A progressive pedagogy for online learning with high-ability secondary school students: a case study. Gifted Child Quarterly, 54, 239-251.

Passow, A. H., Mönks, F. J., \& Heller, K. A. (2010). Research and education of the gifted in the year 2000 and beyond. Em K. A. Heller, F. J. Mönks, R. J. Sternberg, \& R. F. Subotnik (Orgs.), International handbook of giftedness and talent (pp. 883-904). Oxford; Elsevier.

Pérez, S. G. P. B., \& Freitas, S. N. (2013). Do pecado de ser mulher ao medo de ser mulher com altas habilidades/superdotação. Em D. S. Fleith, \& E. M. L. S. Alencar (Orgs.), Superdotados. Trajetórias de desenvolvimento e realizações (pp. 55-74). Curitiba: Juruá.

Prado, R. M. (2010). O talento em uma perspectiva feminina: Características individuais e familiares de pesquisadoras de destaque no Brasil. Dissertação de Mestrado, Universidade de Brasília, Brasília.

Prado, R. M., \& Fleith, D. S. (2012). Pesquisadoras brasileiras: conciliando trabalho, ciência e família. Arquivos Brasileiros de Psicologia, 64, 19-34.

Reis, S. M. (1998). Work left undone. Mansfield Center: Creative Learning Press.

Reis, S. M. (2002), Toward a theory of creativity in diverse creative women. Creativity Research Journal, 14, 305-316.

Reis, S. M. (2005). Feminist perspectives on talent development. Em R. J. Davidson, \& J. E. Davidson (Orgs.), Conceptions of giftedness (pp. 217-245). New York: Cambridge University Press.
Reis, S. M. (2013). Changing paths: Developing creativity and creative minds as a primary goal of gifted education. Palestra proferida na $20^{\text {th }}$ Biennial World Conference of the World Council for Gifted and Talented Children. Louisville, Estados Unidos.

Reis, S. M., \& Sullivan, E. E. (2009). A theory of talent development in women of accomplishment. Em L. Shavinina (Org.), International handbook on giftedness (pp. 487-504). Springer.

Sternberg, R. J. (1986). A triarchic theory of intellectual giftedness. Em R. J. Sternberg, \& J. E. Davidson (Orgs.), Conceptions of giftedness (pp. 233-243). Cambridge: Cambridge University Press.

Sternberg, R. J. (1997). Successful intelligence. New York: Plumen.

Sternberg, R. J. (2005). The WICS model of giftedness. Em R. J. Sternberg, \& J. E. Davidson (Orgs.), Conceptions of giftedness (2a ed., pp. 327-342). New York: Cambridge University Press.

Shavinina, L. (2009a). International handbook on giftedness. Springer.

Shavinina, L. (2009b). A unique type of representation is the essence of giftedness: Toward a cognitive-developmental theory. Em L. Shavinina (Org.), International handbook on giftedness (pp. 231257). Springer.

Subotnik, R., \& Arnold, K. (1995). Passing through the gates: Career establishment of talented women scientists. Roeper Review, 13(3), 14-19.

Vandervert, L. R. (2003). The neurophysiological basis of innovation. Em L. Shavinina (Org.), International handbook of innovation (pp. 17-30). Oxford: Elsevier Science.

Vandervert, L. R., Schimpf, P. \& Liu, H. (2007). How working memory and the cerebellum collaborate to produce innovation and creativity. Creativity Research Journal, 19, 1-18.

Vandervert, L. R. (2009). Working memory, the cognitive funcions of the cerebellum and the child prodigy. Em L. Shavinina (Org.), International handbook on giftedness (pp. 295-316). Springer. 


\section{Sobre a autora}

Eunice M. L. Soriano de Alencar (eunices.alencar@gmail.com)

Doutora em Psicologia, Professora Emérita da Universidade de Brasília, representante do Brasil no World Council for Gifted and Talented Children e membro honorário do Conselho Brasileiro para Superdotação.

A autora agradece à Profa Denise de Souza Fleith e colegas do Grupo de Pesquisa em Criatividade e Superdotação, do Programa de Pósgraduação em Desenvolvimento Humano e Saúde, do Instituto de Psicologia da Universidade de Brasília, pelas numerosas sugestões para o aprimoramento do manuscrito original. 